\title{
Design of an Infusion Rate Control System Based on Single Chip Microcomputer
}

\author{
Qinzhu Wang \\ Xi'an Kedagaoxin University, Xi'an, Shaanxi Province, China
}

Keywords: single chip microcomputer; sensor; stepping motor; can bus; PC; keyboard

\begin{abstract}
Infusion is a common used treatment method in hospitals. Traditional infusion methods have various shortcomings, the infusion speed cannot be controlled, and people need to keep an eye on droplets. Thus, an automatic infusion control device is designed to replace the traditional infusion mode. The purpose of this paper is to control infusion speed through detecting liquid levels in drip tubes through infrared light, and demonstrate the infusion process clearly. The infusion rate can be detected and controlled automatically or manually through the buttons. The integrated communication, namely the control and data transmission between the master station and slave stations is also realized. When abnormal conditions occur, warning light in LED of lower computer will illuminate; electronic communicator will ring. Afterwards, RS232 will response and send the signals to upper computer. When the upper computer receives the signal, the electronic communicator will alarm. The functions of this product must be accurate and stable. The design in this paper theoretically implements expected ideas and points out future research directions and priorities.
\end{abstract}

\section{Introduction}

\subsection{The present situation of this research}

Drip infusion is used to treat patients in many hospitals. According to the survey, patients are usually treated with infusion therapy during hospitalization. The functions of drip infusion are treating illness and conveying nutrition. Patients' ages, disease varieties and drug categories are all factors that influence the speed of infusion. During the infusion process, patients should be accompanied to prevent medical accidents. According to the investigation, many hospitals do not use intelligent infusion equipments now. The traditional infusion method is still employed: hanging the dropping bottle in a high place, then the liquid drips down under pressure. Clamps are used by medical staff to control the dripping speed. It is an inaccurate control method based on nurses' observation and personal experiences; the results may be unreliable, and not conducive to the treatment of patients. At the end of infusion, the phenomenon blood return will appear if the nurse fails to change bottles in time. Blood return can endanger the life of the patient if it is not stopped in time. At the same time, medical staffs need to regularly check patients' situation, which increase their workload and may lead to contradiction between nurses and patients. Therefore, after research, an intelligent infusion monitoring system is designed to help patients and reduce the workload of medical staffs.

Not long ago, automated infusion devices were widely used in hospitals. It can not only detect the infusion speed very accurately, but also alarm and prevent blockage. In our country, only large hospitals are able to purchase this kind of equipment. Most of these machines are imported, since foreign devices are in variety and have good performances. Now the research and development of the infusion device alarm system has become the focus of people's attention. It is known from relevant survey that there are 58 kinds of transfusion alarm techniques in China. But there are some problems in these techniques, including problems about safety, performance and cost. Few of them can become professional techniques. As medical equipments, the realization, timeliness and security of these machines must be guaranteed. Devices are of design significance if they can be applied to every patient. These devices must be easy to operate and low cost. The smooth promotion could be realized 
on the basis of safety, reliability, as well as operation and cost guarantee.

According to the survey, due to the large population of China, the number of hospital patients in China is larger than that of other countries; medical devices in our country also increase rapidly in recent years. At present, China cannot fully realize the monitoring of automatic infusion. Therefore, it is particularly urgent to develop a device that can automatically monitor the infusion speed.

\subsection{The significance of this research}

With the increasing number of patients, the medical industry has developed continuously. In the aspect of medical monitoring, added medical equipments must be diversified, automatic and intelligent. With the continuous development of science and technology, a great development opportunity and platforms has been created for the medicine industry. Because of the diversification of patients, current medical technology and equipments cannot solve all the problems in hospital. Therefore, medical equipments based on network technology enjoy great development and market potential.

Drip infusion is the most common used medical means. But there are some deviations in the control and detection of infusion speed. Medical staffs control the infusion speed according to their experiences and observation. The operation is tedious, inaccurate and may be harmful to patients. If we can develop a dripping speed control device, it will become popular very quickly. The current infusion control devices are expensive and inconvenient to carry, and increase the expenditure of patients. In that case, we design and develop an infusion speed controlling device based on single chip microcomputer. Data required by doctors are provided by this device, which improves the effectiveness and timeliness of illness treatment. The system is divided into infusion detection device and control device, which is conducive to the remote monitoring of patients. Multiple bedding control devices send out alarm signals simultaneously when the transfusion situation changes. The device is simple and easy to carry, and can be applied to different hospital departments. The device has many advantages: high stability, easy installation and low cost. Therefore, the design and development of the infusion speed control system is beneficial to patients and our country.

\subsection{Main contents of this research}

This design is mainly based on AT89C52 microcontroller, and supplemented by ULN2003 chips. The design combines multiple techniques and develops a new type infusion device, including embedded programming technology, single chip control, as well as communication and stepping motor control techniques. The main functions of this system are: (1) setting the speed required for infusion; (2) automatically adjust the flow of liquid in the drip tube; (3) automatically send out alarm signals when the infusion is completed or the transfusion situation changes. The main features of the system are as following. (1) The stepping motors are evenly operated and can be positioned accurately. (2) The interference of infrared tube can be prevented by the means of modulation. (3) The design is reasonable and easy to operate.

\section{Hardware Design for Slave Stations}

\subsection{Block diagram of the slave station and the core circuit}

A block diagram is designed according to the functions of lower computer system.

The lower computer system uses AT89C52 single chip microcomputer as the CPU, and uses electronic signal and light emitting diodes to realize sound and light alarm. The stepping motor is controlled by the method of four phase and eight beat.

AT89C52 MCU is a controller with low power consumption and high function, and has 8K programming Flash memorizer in system. In addition to programming on conventional programmers, Flash chip allows program memorizer to program in the system. AT89C52 can be adjusted to 0HZ state for static logic operation, and there are two power saving modes for choice. The idle mode is one of them. Its price is cheap; an inside watchdog software is enough for the memorizer to program smoothly in the system. It is very appropriate for AT89C52 to become the CPU of slave stations. 


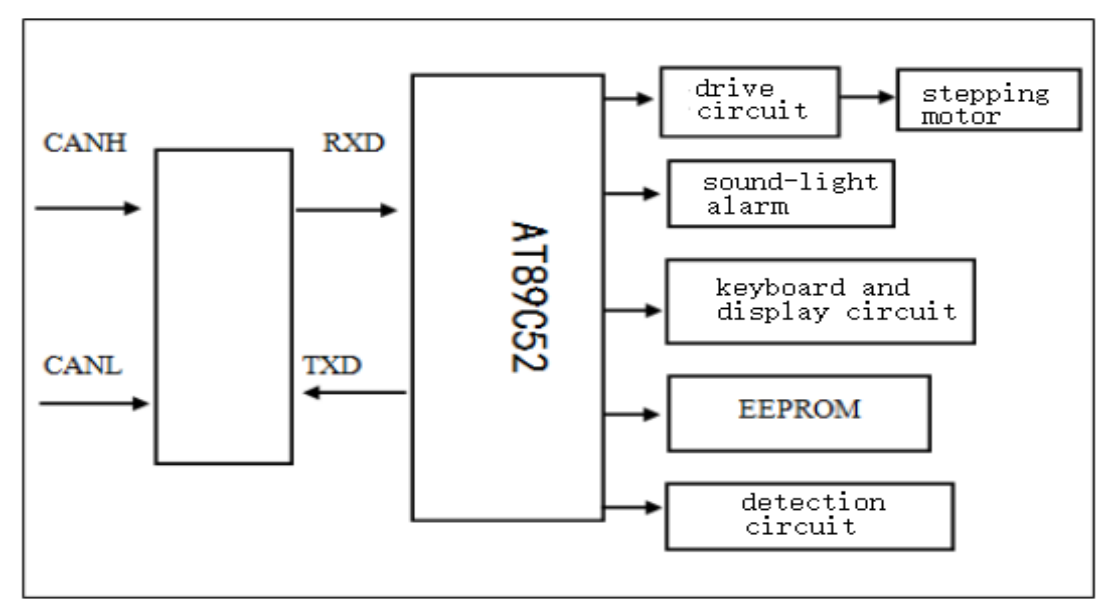

Figure 1. Structure diagram of the slave station system

\subsection{Design of each unit in slave stations}

The station has many subsystems. Detailed circuit principles cannot be displayed in the circuit diagram. It is necessary to carefully analyze the working principles of each module.

Drop signal detection unit. The most important part of designing is the dripping speed detection circuit. If we want to achieve dripping speed control, we must detect the data accurately.

The process of transfusion drop detection is based on infrared light detection technology; photoelectric detector is used to detect the dripping speed. After the photoelectric induction infrared light device emits red light, the light is irradiated on the receiving diode through the plastic dropper. It converts the received light signal into current signal and sends it to the single chip microcomputer. If there is no drop in the tube, the light will easily penetrate the plastic dropper; direct transmission will lead to stronger light. At that time, current signal converted by the diode is stronger. If the current signal drops, the absorption and reflection will obstruct the penetration of light. Light and electrical signal will become weaker. Finally, current signal transforms into voltage signal through photosensitive resistor; the infusion speed is calculated through the extraction of single chip microcomputer.

Keyboard and display unit. This design defines the functions of keys.

Key 1: click once represents selecting the volume of liquid in the bottle, and then press the button once again to conform; press two times represent selecting the type of infusion tube, and then press the button once again to conform.

Key 2: selecting 500 milliliters; selecting 20 drops / milliliter

Key 3: selecting 250 milliliters; selecting 15 drops / milliliter

The display unit adopts LCD; LCD has following notable characteristics.

(1) low voltage and less consumption

(2) flat plate structure

(3) relatively passive display

(4) enough information displayed

(5) no electromagnetic radiation

(6) long service time

The aim of this paper is to realize the detection and control of one-to-one remote transmission system; a device which can collect remote monitoring information and upload it to the master station is needed. The RS-485 serial interface is used to connect the lower and upper computers effectively, in order to achieve the transmission of right data and improve efficiency. The connection between the lower computer and the PC master station can be realized through DNC bus; data is transmitted to the single chip microcomputer. In this project, the serial converter and data bus are used together, which can not only help to achieve the transfusion functions, but also reduce design difficulty. Through RS232 serial port and field bus, data information is transformed; an intelligent network monitoring system is formed. 
The RS232 serial interface in upper computer can be transformed into a serial converter bus through different types of converters. The lower computer and the bus are bridged by RS-232 serial interface chip. All lower computers have automatic recognition functions. When the system starts to work, each lower computer identifies its own IP address. They cannot interact with each other. But as long as the upper computer operates, slave stations monitor with the control of upper computer. If the upper computer sends execution instructions, lower computers will begin to execute immediately. Lower computers compare the addresses they received with their own addresses. If the address is correct, instructions will be followed. Otherwise, they will stop. The design of node circuits is the most important part in communication hardware circuit. Communication node circuits are composed of following parts: (1) RS-232 serial interface circuit; (2) single chip microcomputer circuit; (3) power circuit. The main function of RS232 interface circuit is to connect different devices for convenient communication.

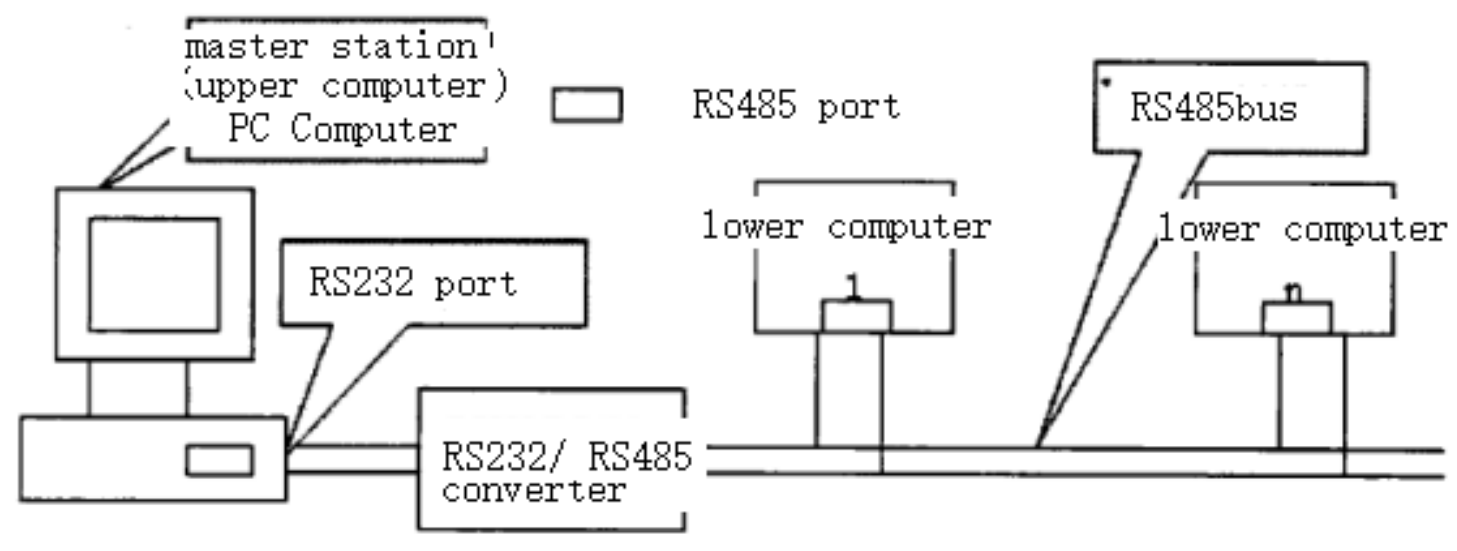

Figure 2. CAN bus communication system for infusion monitoring

\section{Conclusion}

The rapid development of modern medical technology requires better medical facilities and services. Infusion is one of the most common treatment methods in our life, and its monitoring equipment has become more and more popular. The infusion control system should be developed in the direction of intelligence, automation and high end. But at present, this kind of infusion control devices cannot be found in general wards of many hospitals. Under that situation, a set of infusion control system with advantages of low price, simple operation and high reliability will attract peoples' attention and become extremely popular. In this paper, an infusion monitoring system based on AT89C52 microcontroller is designed to meet the needs of nurses and patients. The system can not only reduce the workload of medical staffs, but also prevent medical accidents caused by negligence. The design in this paper greatly improves the work efficiency of nurses and has great significance.

The infusion control system is designed to realize the automatic, accurate and non-contact functions of the whole process, and improve the traditional infusion monitoring mode. Based on optical effects of the liquid drop, an infusion counting device is designed by using the infrared light sensor. The fact shows that the infusion status can be judged by the drop counter.

\section{References}

[1] X.F. Liu, Development of intelligent infusion sets, J. Science \& Technology Information. 4 (2010).

[2] M. Zhang, S.T. Wang, C.Y. Yu, M.Y. Zhang, The development tendencies of infusion pumps/syringe pumps, J. Chinese Journal of Medical Lnstrumentation. 33 (2009).

[3] Y. Chen, X. Wang, Design of intelligent infusion monitor and control system based on 
photoelectric technology, J. Nuclear Electronics. 29 (2011).

[4] Y.Q. Gao, Principle and Application of Single Chip Microcomputer and C51 Programming Technology, China Machine Press, Beijing, 2011.

[5] D.M. Zhang, Principle and Application of Single Chip Microcomputer, Higher Education Press, Beijing, 2010.

[6] Q. Li, G.S. Liang, Design and implementation of an infusion monitoring system model based on MCGS, North China Electric Power University, 2010

[7] Z.T. Wang, R.F. Wang, T.F. Yan, Intelligent infusion speed monitor, J. Automation and instrumentation. 5 (2010).

[8] W. Shan, Design and implementation of the infusion monitoring system based on AT89S52, Hefei University of Technology, 2009

[9] Z. Li, C.J. Yang, W. Zheng, Software and hardware design of venous transfusion monitor in medical treatment, J. Applied Science and Technology.28 (2011).

[10] Y. Zhang, Design of infusion monitoring and control system, Dalian Jiaotong University, 2006

[11] H.T. Li, X. Zhao, X. Li, J.K. Xia, Development of intelligent infusion monitoring system for transfusion, J. Journal of Shenyang University of Technology. 28 (2012).

[12] Y. Jiang, Research on remote monitoring system of infusion based on AT89C51, Kunming University of Science and Technology, 2009.

[13] F.G. Bai, W. Bai, Design of Transfusion Speed Monitor Based on MCU and Photoelectric Switch, J. International Conference on Information Engineering and Computer Science. 4 (2009).

[14] T.A. Neff, J.E. Fischer, G. Schulz, O. Baenziger, M. Weiss, Infusion pump performance with vertical displacement: effect of syringe pump and assembly type, J. Intensive Care Medicine. 27 (2011). 